\title{
The Growth and Respiration of Bacterial Colonies
}

\author{
By JULIAN W. T. WIMPENNY AND MARY W. A. LEWIS \\ Department of Microbiology, University College, \\ Newport Road, Cardiff CF2 ITA
}

(Received 20 June 1977)

\begin{abstract}
Young colonies of two swarming organisms, Bacillus subtilis and Proteus vulgaris, grew about as quickly on solid media as in liquid culture whilst four non-swarming organisms, Bacillus cereus, Enterobacter cloacae, Escherichia coli and Staphylococcus albus, all grew slower on solid than in liquid media. Oxygen uptake by young colonies of B. subtilis, followed manometrically, increased exponentially at about the same rate as unrestricted aerobic growth. All other colonies demonstrated accelerating respiration which was either not strictly exponential or, in the case of $S$. albus, definitely biphasic, with a fast then a slow exponential rate of increase. Actual and potential respiration was determined for each species by measuring oxygen uptake before and after resuspending the colony in liquid medium. The ratio of actual to potential respiration was largest in the flat, spreading B. subtilis and smallest in the small, hemispherical $S$. albus. Calculations suggest that oxygen penetrates between $3 \mathrm{I}$ and $4 \mathrm{I} \mu \mathrm{m}$ into colonies of B. cereus, Ent. cloacae and $E$. coli and only $9 \mu \mathrm{m}$ into colonies of $S$. albus.
\end{abstract}

\section{INTRODUCTION}

The relationships of different bacteria within a colony are ill understood. Greene (1938) showed that certain anaerobic species sporulated in the surface layers of colonies exposed to air. Legroux \& Magrou (1920) showed that only cells in the surface layers of colonies of Vibrio cholerae picked up a vital stain and were probably alive, whilst those beneath were an amorphous mass with no recognizable structures.

Pirt (1967) reported the radial growth rate of colonies of Enterobacter aerogenes, Escherichia coli and Streptococcus faecalis to be linear with time. Theoretically, growth, including this parameter, should obey an exponential relationship. Pirt explained the discrepancy in terms of diffusion of nutrients to the population: only at the edge of the colony are substrate and oxygen present in excess together.

Cooper, Dean \& Hinshelwood (I968) showed that in very old colonies linear increase in radius gives way to a linear increase in area and this is explained as being due to removal of nutrient from the agar in front of the expanding colony, an interpretation that seems to fit the observations of Rieck, Palumbo \& Witter (1973), who measured the concentration of glucose in the agar used to grow Pseudomonas fluorescens.

Palumbo et al. (1971), regarding the colony (also of Pseudomonas fuorescens) as domeshaped like a slice taken off a regular sphere, showed that colony volume increases exponentially over the first day's growth, although the viable count does not. Colonies on a simple defined medium are about $100 \mu \mathrm{m}$ high, whereas on rich media they are $600 \mu \mathrm{m}$ high.

This paper reports the growth, overall respiration and potential respiration rate of colonies of six diverse species of bacteria. 


\section{METHODS}

Organisms. The six micro-organisms used were Bacillus subtilis NCIB8059, Bacillus cereus, Enterobacter cloacae, Proteus vulgaris, Escherichia coli $\mathrm{KI} 2$ and Staphylococcus albus. The stock cultures were grown on Dorset egg slopes (Oxoid) and kept at $4{ }^{\circ} \mathrm{C}$. Subcultures to new medium were made at two-monthly intervals. The medium used for colony growth experiments was Oxoid Tryptone Soya Broth plus $I \cdot 2 \%(\mathrm{w} / \mathrm{v})$ Difco Bacto-agar. Bacillus subtilis and B. cereus were grown at $30^{\circ} \mathrm{C}$, whilst $P$. vulgaris, E. coli, Ent. cloacae and $S$. albus were grown at $37^{\circ} \mathrm{C}$. All sterilizations, unless otherwise stated, were performed at $12 \mathrm{I}^{\circ} \mathrm{C}$ for I $5 \mathrm{~min}$ in a pressure cooker.

Growth. Growth was measured as extinction at $550 \mathrm{~nm}$ in a Pye Unicam SP600 spectrophotometer and related to dry weight through a calibration curve for each organism.

Preparation and inoculation of Petri dishes. Sterile Tryptone Soya Broth agar $(25 \mathrm{ml}$ in each Petri dish) gave a layer of agar about $3.6 \mathrm{~mm}$ thick. Dishes were dried, open and inverted, at $37^{\circ} \mathrm{C}$ for $\mathrm{I} \frac{1}{2} \mathrm{~h}$, or for $45 \mathrm{~min}$ for swarming cultures of $B$. subtilis, and were then stored at $37^{\circ} \mathrm{C}$ inverted in an airtight container over a layer of water so that they were in a humid atmosphere for 8 to $15 \mathrm{~h}$ before inoculation. All subsequent incubation was in the same water-saturated environment. Inoculations were made using a Pasteur pipette drawn out to a fine sterile point. This was stabbed once into a lawn plate of the inoculating organism, then into the plate being seeded.

Liquid culture. Each species was grown in Tryptone Soya Broth shaken in $250 \mathrm{ml}$ Erlenmeyer flasks at $37^{\circ} \mathrm{C}$.

Respirometry. A Gilson differential respirometer (Gilson Medical Electronic, 69 Rue Gambetla, Villeres Le Bel (S. et. O.), France) was used.

Manometer flasks without centre wells with a strip of fluted filter paper in the side-arm were air-sterilized and plugged. Sterile Tryptone Soya Broth agar $(4 \mathrm{ml}$ in each flask) gave an agar depth of about $3.6 \mathrm{~mm}$. The cotton-wool plugs in the tops and side-arms were covered with Parafilm self-sealing tape and the flasks were stored in the humid container in the $37^{\circ} \mathrm{C}$ incubator for $\mathrm{I} 2$ to $18 \mathrm{~h}$ before use. Immediately before inoculation, which was exactly as described for plates, $0.2 \mathrm{ml}$ sterile $2 \mathrm{M}$-potassium hydroxide was added to the side-arm. Two flasks were left uninoculated each time as controls. The flasks were then attached to the respirometer using sterile lanolin to grease the side-arm stoppers and necks of flasks. The respirometer apparatus was left open to the atmosphere for 5 to $6 \mathrm{~h}$. Half an hour before respiration measurements were started the Gilson respirometer was closed to the atmosphere and allowed to equilibrate, while shaking, for $10 \mathrm{~min}$ before the manometer valves were closed and readings commenced. It is assumed in colony respiration measurements that increases in cumulative oxygen uptake rates are directly proportional to increases in oxygen demand. Within the limits of accuracy of these experiments this assumption is justified.

To estimate the potential respiration of cells in the colony, $2 \mathrm{ml}$ sterile Tryptone Soya Broth was added to one or more flasks which were then allowed to equilibrate, while shaking, for $5 \mathrm{~min}$ before readings were taken at $\mathrm{I}$ or $2 \mathrm{~min}$ intervals for a total of $20 \mathrm{~min}$ after adding the Tryptone Soya Broth. $E_{550}$ was then measured against a Tryptone Soya Broth blank.

Colony dimensions. Petri dishes were inoculated with I to 5 colonies each. At timed intervals, visible colonies were measured with a transparent ruler under a Zeiss binocular microscope viewing the colony through the agar of the inverted Petri dish. The mean of two colony diameters at right angles was taken for each colony.

At timed intervals, some colonies were washed off the Petri dishes with sterile Tryptone Soya Broth, made up to a standard volume with broth, mixed and homogenized; the $E_{550}$ of the suspension was measured against a Tryptone Soya Broth blank.

Oxygen penetration into bacterial colonies. Approximate estimates of oxygen penetration into the colony were based on the ratio of actual to potential respiration rates.

The following assumptions were made. (i) That the shape of a colony of non-spreading bacteria is a segment of a sphere (as suggested by Palumbo et al., 1971). This suggestion was confirmed for each species by surveying some colonies using a microscope with a calibrated vernier focus to observe different parts of the surface of the colony or, if this was not clear, a very fine layer of talcum powder sprinkled on the colony. (ii) That the organism's dry weight was one-quarter of its wet weight. (iii) That the density of cell material approximates to $\mathrm{I} \cdot \mathrm{I} \mathrm{g} \mathrm{cm}^{-3}$.

The formula for a segment of a sphere is

$$
V_{\mathrm{t}}=\frac{\pi}{6} h\left(3 r^{2}+h^{2}\right)
$$


where $V_{\mathrm{t}}$ is the total colony volume, $h$ its height and $r$ its base radius. This formula can be rearranged as a cubic equation in $h / r$ :

$$
(h / r)^{3}+3(h / r)-\frac{6 V_{t}}{\pi r^{3}}=0
$$

An approximate value for $h$ was obtained by the method of false position and this approximate value was then used to solve equation 2 by the Newton-Raphson method.

Average values for the ratio of actual to potential respiration rates were determined from respiration studies. These values, applied to the total colony volume, led to estimates of the aerobic volume of the colony.

The anaerobic centre of the colony was also assumed to approximate to a segment of a sphere with a volume $V_{\mathrm{a}}$ defined by equation I where $(h-d)$ and $(r-d)$ were substituted for $h$ and $r, d$ being the thickness of the outer aerobic shell and so equal to the depth of oxygen penetration. Thus the volume of the aerobic surface layer $\left(V_{0}\right)$ is, by difference,

$$
V_{0}=\frac{\pi}{6}\left[3(h+r)^{2} d-6(h+r) d^{2}+4 d^{3}\right]
$$

which yields a cubic equation in $d$ :

$$
4 d^{3}-6(h+r) d^{2}+3(h+r)^{2} d-\frac{6 V_{0}}{\pi}=0
$$

Equation 3 was also solved by the method of false position followed by the Newton-Raphson method.

\section{RESULTS AND DISCUSSION}

\section{Growth in liquid and on solid media}

The data from both liquid and solid cultures were plotted semilogarithmically (Fig. I). Straight lines with statistically significant regression coefficients were plotted in all cases. Early colonial growth, measured as an increase in dry weight, is thus exponential, although (except in two cases) it is considerably slower than growth in liquid cultures. The two exceptions, $B$. subtilis and $P$. vulgaris (Fig. I $a, c$ ), are both swarming organisms which suggests that this phenomenon avoids some of the restrictions imposed by diffusion of nutrients from the agar and of oxygen from the atmosphere. Comparative growth rates from each method of determination are shown in Table $\mathrm{I}$.

\section{Respiration rate of intact bacterial colonies}

The increase in colony respiration rate was plotted both arithmetically and semilogarithmically (Fig. 2). The data indicate that respiration increases in an accelerating fashion which is not necessarily logarithmic. During early stages of growth the colony is small enough to allow nutrients and oxygen to diffuse freely to all cells, which should be respiring at their maximum $Q_{\mathrm{o}_{2}}$ value as the colony grows exponentially in an unrestricted fashion. If the colony can swarm so that cells grow in regions containing excess nutrient and if it remains sufficiently flat to allow oxygen to penetrate to all cells, then the increase in cell mass and in respiration should continue at the same early exponential rate. This appeared to be true for B. subtilis (Fig. $2 a$ ) in which, after an early lag, respiration increased exponentially. This rate, expressed as a respiration doubling time, is similar to the mass doubling time obtained in the growth experiments. Later in the growth phase the increase slowed down, presumably as the colony thickened and encountered diffusion problems.

The other swarming organism, $P$. vulgaris (Fig. $2 c$ ), showed an early fast rate of increase in respiration followed by a slower, nearly exponential increase. This suggests that factors other than oxygen accessibility influenced the respiration rate as the doubling time for increase in respiration was less than the mass doubling time observed in liquid culture and on solid medium. However, these results indicate that there was little, if any, restriction due to oxygen limitation in the young colonies of swarming organisms, which spread rapidly and remained relatively flat until late in the growth phase. 

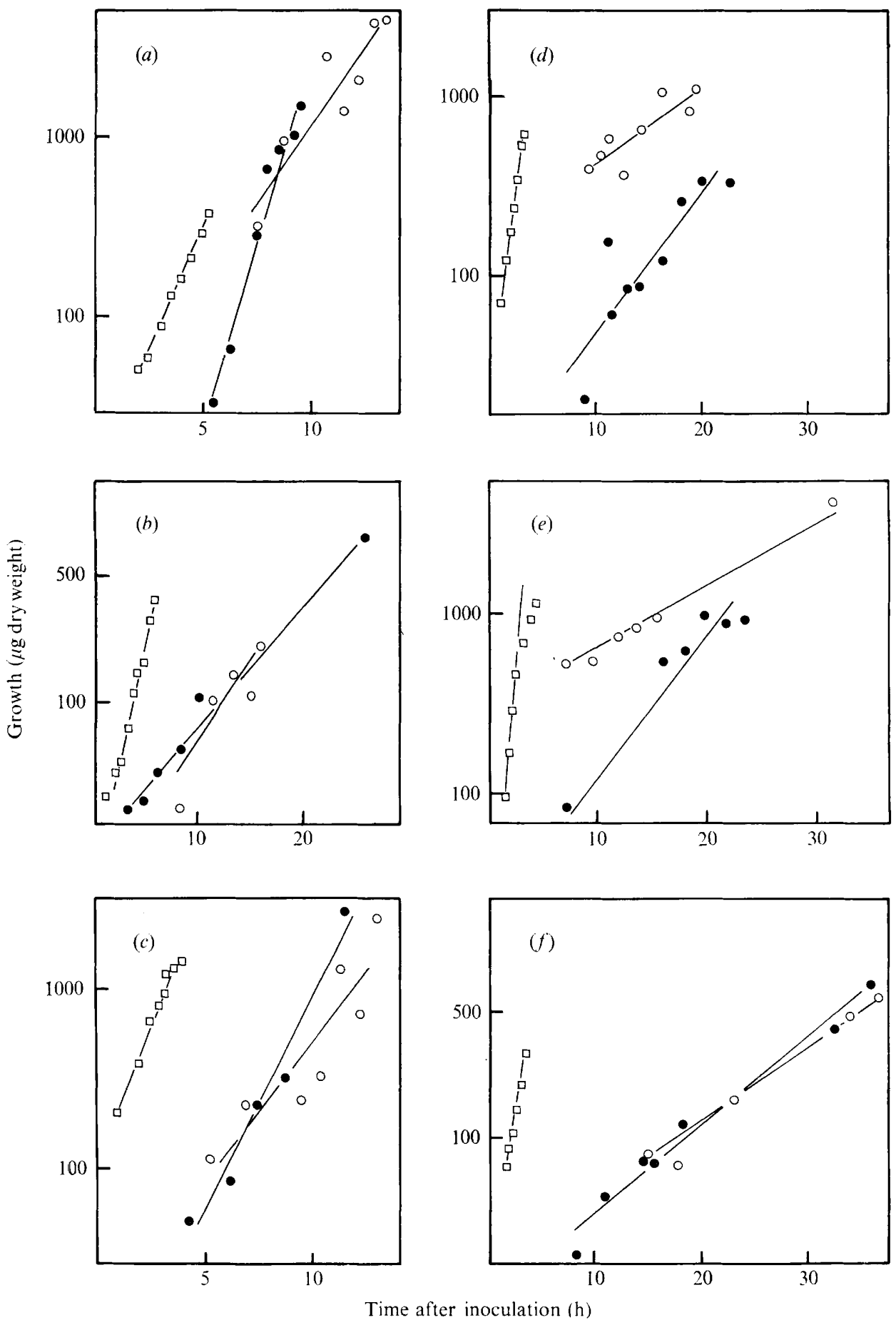

Fig. I. For legend see facing page. 
Table I. Specific growth rate of each species grown in liquid medium or in the same medium solidified with agar

Specific growth rates $(\mu)$ are expressed in $\mathrm{h}^{-1}$.

Organism

Swarmers

B. subtilis

P. vulgaris

Non-swarmers

B. cereus

E. coli $\mathrm{KI} 2$

Ent. cloacae

S. albus

Liquid culture
(dry weight
increase)

0.63

0.63

0.69

0.92

$\mathrm{I} \cdot 38$

0.81
Plate colony
(dry weight
increase)

0.99

0.50

0.1 6

0.17

0.19

0. I I
Flask colony

(dry weight

increase)

0.39

0.20

0.05

0.04

0.06

The diffusion of oxygen and nutrients into the colony of a non-swarming organism becomes restricted much earlier in growth as the height of the colony increases. All the non-swarmers examined are facultative anaerobes so, given sufficient nutrient, the centres of these colonies would grow anaerobically whilst the outer layers continue to grow at the faster aerobic rate. The increase in total mass would then occur at a much slower exponential rate than in fully aerobic liquid cultures.

Except in the very early stages of growth, E. coli (Fig. 2d) and Ent. cloacae (Fig. 2e) showed an increase in respiration that was close to exponential, as did $S$. albus (Fig. $2 f$ ), although with $S$. albus the initial, very rapid rate of increase changed over a short period to a slower, though still approximately exponential rate. The respiration doubling times for these three species were much slower than the aerobic mass doubling times in liquid culture. It seems likely that these colonies are demonstrating exponential growth with the central core growing fermentatively whilst the outer skin grows aerobically. Since the aerobic growth is faster and gives a greater yield than the anaerobic growth, the surface layers can be expected to develop more quickly until nutrient diffusion limits the growth at the upper surface of the colony so that only at the edge of the growing colony will oxygen and nutrients be in excess. Thus the increase in radius of the colony will be largely due to the growth of aerobically-adapted cells.

Bacillus cereus (Fig. $2 b$ ) always showed a constantly falling rate of increase in the semilogarithmic plot. This suggests a gradual transition from predominantly aerobic to predominantly anaerobic metabolism as the colony grows.

\section{Actual and potential respiration rates}

Actual and potential respiration rates, determined as described in Methods, are shown in Fig. 3 and the ratios of actual to potential rates are shown as histograms in Fig. 4. The potential respiration rates measured are for cells derived from the lower anaerobic regions as well as from the aerobically-adapted surface layers of the colony. However, since a fully aerobic colony of this size is, by definition, impossible to obtain, it was felt that

Fig. I. Growth of each species in aerated liquid Tryptone Soya Broth and on Tryptone Soya Broth agar. Growth was determined turbidimetrically and data are plotted logarithmically. $O$, Growth of colonies on the surface of Tryptone Soya Broth agar plates; the colonies were washed off the plates with Tryptone Soya Broth and gently homogenized before measurement. , Growth of colonies on the surface of Tryptone Soya Broth agar in Gilson respirometer flasks incubated, with shaking, in the respirometer bath; the colonies were dispersed by shaking with Tryptone Soya Broth before measurement. $\square$, Growth in aerated liquid Tryptone Soya Broth. (a) B. subtilis at $30^{\circ} \mathrm{C}$; (b) B. cereus at $30^{\circ} \mathrm{C} ;\left(\right.$ c) P. vulgaris at $37^{\circ} \mathrm{C} ;($ d $)$ E. coli $\mathrm{KI} 2$ at $37^{\circ} \mathrm{C}$; (e) Ent. cloacae at $37^{\circ} \mathrm{C} ;(f)$ S. albus at $37^{\circ} \mathrm{C}$. 

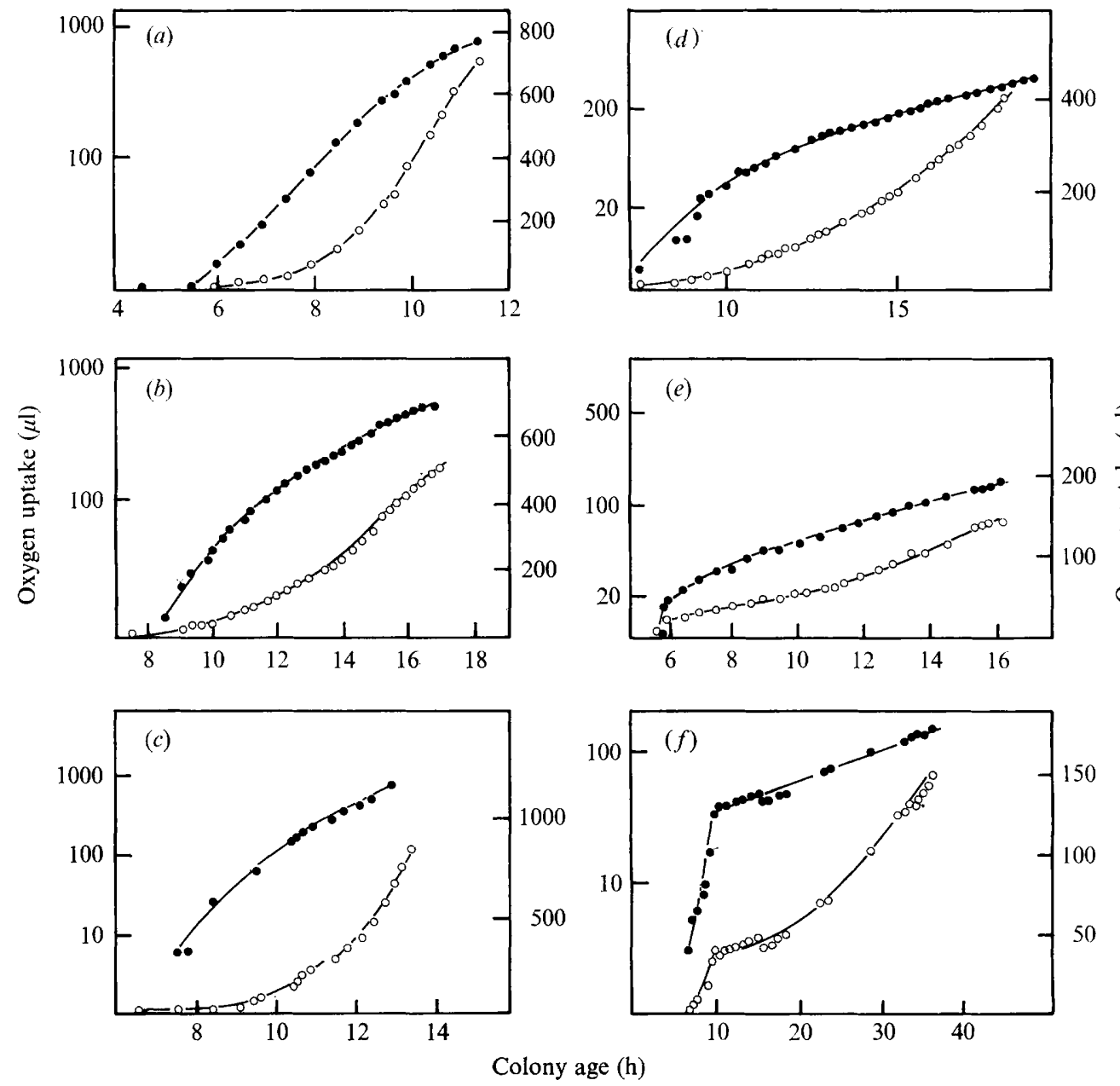

Fig. 2. Oxygen uptake by individual colonies of each species grown on solid medium in respirometer flasks. The data are plotted arithmetically $(O$, right-hand ordinate scale) and logarithmically (O, left-hand ordinate scale). (a) B. subtilis; (b) B. cereus; (c) P. vulgaris; (d) E. coli KI $;(e)$ Ent. cloacae; $(f)$ S. albus.

our procedure would give a closer measure of the potential respiration than could be gained, for example, from an equivalent biomass of aerobic cells produced separately in a liquid culture, when more complicated assumptions would have to be made.

The Gram-positive swarmer, B. subtilis, which speedily covers the agar surface with a thin opaque layer, had actual respiration rates that nearly equalled the potential respiration value, suggesting that, for the most part, this swarmer is not oxygen limited - presumably because of the thinness of the spreading colony.

The other Gram-positive aerobe, B. cereus, had the next highest actual/potential respiration rate with an actual respiration rate of just under half the potential value. It may be that the corrugations on the surface of this shallow dome-shaped colony allowed a greater surface area for oxygen penetration than if it were smooth. Actual/potential respiration values were less useful when applied to colonies of $P$. vulgaris. Inspection of the graph (Fig. $3 c$ ) suggests that young thin colonies had only slight oxygen diffusion problems but these became more severe as the colony became thicker. The mean figure suggests that only about one-quarter of the cells in the colony were able to respire. 

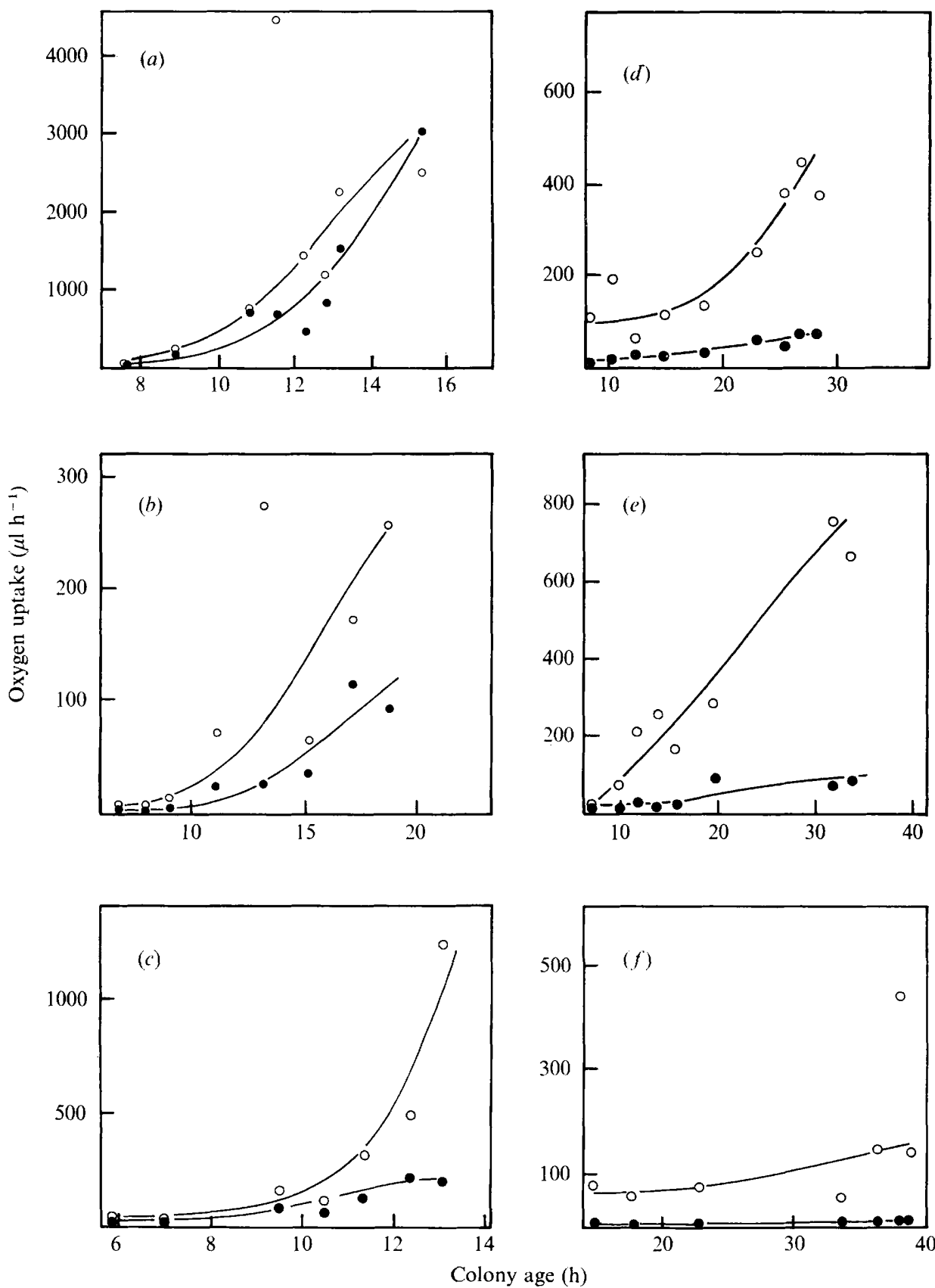

Fig. 3. Actual (O) and potential (O) respiration rates of colonies of each species grown on solid medium in respirometer flasks. The actual respiration rate was determined just before the addition of $2.0 \mathrm{ml}$ Tryptone Soya Broth which disperses the colony. The potential respiration rate was determined as soon after this addition as possible and before there was any significant growth. (a) B. subtilis; (b) B. cereus; (c) P. vulgaris; (d) E. coli $\mathrm{KI} 2$; (e) Ent. cloacae; $(f) S$. albus. 


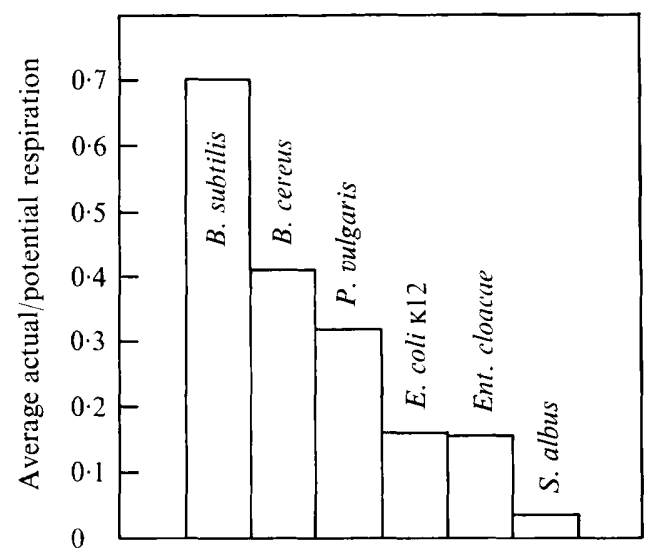

Fig. 4. Average ratio of actual to potential respiration rates for each species.

The two non-swarming enteric organisms, E. coli and Ent. cloacae, which both have smooth surfaces, had reasonably low values for actual/potential respiration indicating that only about one-tenth of the cells of the colony are respiring aerobically. The nearly hemispherical colonies of the Gram-positive coccus $S$. albus have the smallest surface area to volume ratio of the organisms used. This is reflected by the low values for actual/ potential respiration which show that only about one-twentieth of the cells of the colony are respiring aerobically.

\section{Oxygen penetration into bacterial colonies}

If the flow of nutrients and oxygen into the colony were unrestricted and growth inhibitors did not accumulate, then colony growth could be expected to be exponential. The data show that mass increase is logarithmic but that increase in respiration is usually not truly exponential suggesting that as the colony matures a smaller proportion of the cells are respiring aerobically.

Pirt (1967) has calculated oxygen penetration by applying the diffusion equation and using concepts of colony growth based on the study of populations of organisms in submerged homogeneous cultures. He obtains a value of $40 \mu \mathrm{m}$ in colonies of $E$. coli, using the diffusion coefficient of oxygen through water.

It is assumed in this paper that the ratio of actual to potential respiration rates reflects the fraction of the colony having sufficient oxygen to respire aerobically. This ignores any physiological changes occurring in cells in different regions of the colony due to starvation, lysis and the accumulation of toxic materials but these problems appear to be insignificant with the young colonies used. Adaptation to aerobiosis or anaerobiosis may have slightly more effect but the $Q_{\mathrm{O}_{2}}$ for glucose (the major carbon source in Tryptone Soya Broth) is similar in bacteria growing aerobically or anaerobically (Wimpenny, 1969).

The depth to which oxygen penetrates into non-swarming bacterial colonies was estimated as outlined in Methods. The results of these calculations (Table 2) indicate a mean value of $3 \mathrm{I} \mu \mathrm{m}$ for $E$. coli, $4 \mathrm{I} \mu \mathrm{m}$ for Ent. cloacae and $37 \mu \mathrm{m}$ for $B$. cereus. These results are similar to the $40 \mu \mathrm{m}$ calculated as the depth of oxygen penetration into growing colonies of $E$. coli by Pirt (1967), using growth kinetics determined in liquid culture. However, for $S$. albus a much lower value of $9 \mu \mathrm{m}$ was obtained for the depth of oxygen penetration into colonies. It is unlikely that this low value is due to a very high $Q_{\mathrm{o}_{2}}$ for surface cells, since published data do not indicate high $Q_{\mathrm{o}_{2}}$ rates for staphylococci. It seems more likely that it reflects a lower diffusion rate of oxygen through and between the tightly-packed 


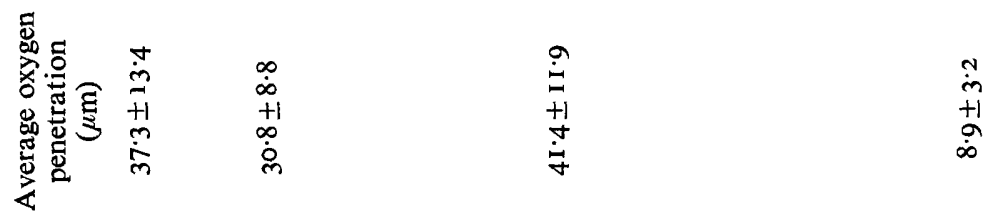

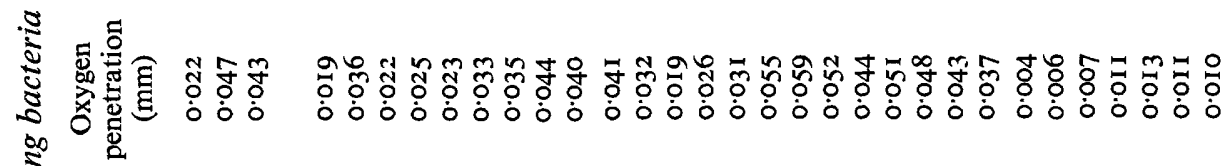

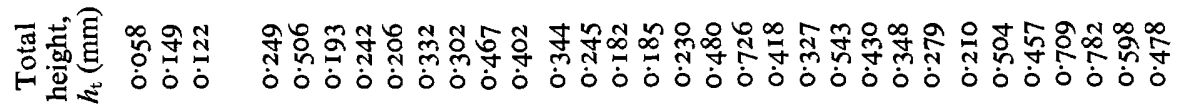

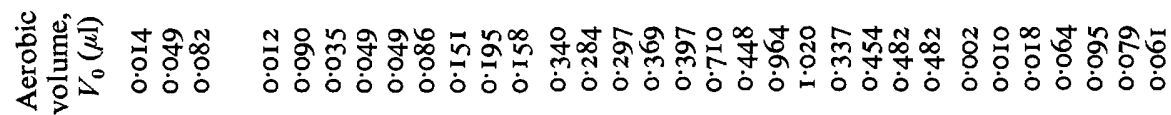
$\stackrel{8}{.5}$

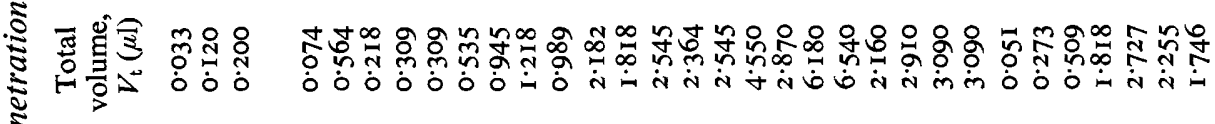

$\lesssim$

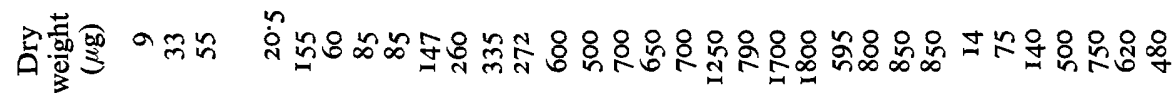
bे

$\frac{\sqrt[2]{2}}{\sqrt[3]{2}}$

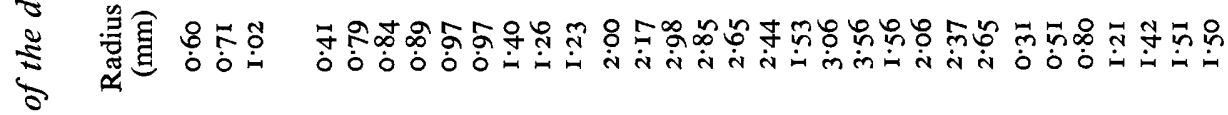

苟

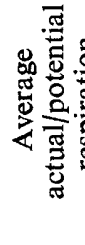

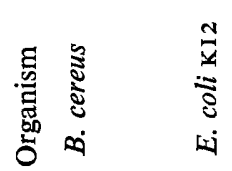

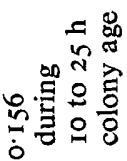

ํ. 
spherical cells of this species than through the more loosely-packed cells of the other three species.

The bacterial colony is clearly a complicated structure whose morphology depends upon a number of factors including the shape of the cell, extracellular structures, such as locomotor appendages or capsular material, and on the mode of reproduction of the cell. The growth of cells and final organization of the colony will also be affected by diffusion. Thus there are gradients of nutrients diffusing upwards from the agar surface, of metabolic products which diffuse outward from the colony, and of atmospheric constituents diffusing into and out of the structure. This work has attempted to show that not only does the oxygen diffusion gradient affect the rate of growth of the organism but that colonial morphology greatly influences the oxygen relationships of the structure and, in particular, that the swarming behaviour shown by some organisms does much to circumvent oxygen limitation.

In calculating oxygen penetration the authors gratefully acknowledge help and advice given by W. T. Coakley in our own department and M. T. Heydeman from the Department of Microbiology, University of Reading.

\section{REFERENCES}

Cooper, A. L., Dean, A. C. R. \& Hinshelwood, C. (1968). Factors affecting the growth of bacterial colonies on agar plates. Proceedings of the Royal Society Br7r, 175-199.

GreENE, H.C. (1938). Colony organisation of certain bacteria with reference to sporulation. Journal of Bacteriology 35, 26I-273.

Legroux, R. \& MAGrou, J. (1920). Etat organisé des colonies bactériennes. Annales de l'Institut Pasteur 34, 4I 7-433.

Palumbo, S. A., Johnson, M. G., Rieck, V. T. \& WITTER, L. D. (197I). Growth measurements on surface colonies of bacteria. Journal of General Microbiology 66, I37-I 43 .

PIRT, S. J. (1967). A kinetic study of the mode of growth of surface colonies of bacteria and fungi. Journal of General Microbiology 47, I8I-197.

Rieck, V. T., Palumbo, S. A. \& Witter, L. D. (I973). Glucose availability and the growth rate of colonies of Pseudomonas fluorescens. Journal of General Microbiology 74, I-8.

WimpenNy, J. W. T. (1969). Oxygen and carbon dioxide as regulators of microbial growth and metabolism. Symposia of the Society for General Microbiology I9, 16I-197. 